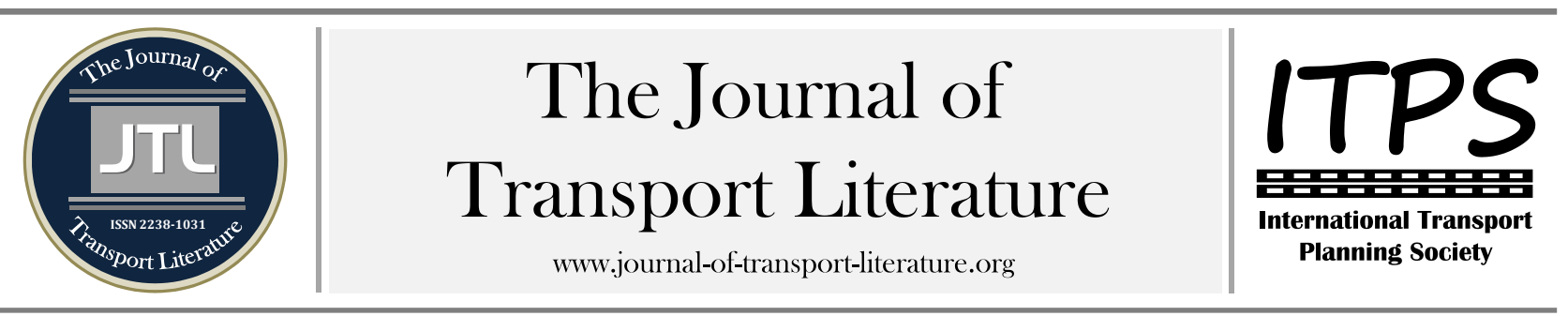

\title{
Custo de transporte e alocação da demanda: análise da rede logística de uma produtora brasileira de fertilizantes nitrogenados
}

Alessandra Andrade Pereira1,+; Murilo Alvarenga Oliveira²; Ilton Curty Leal Júnior²

${ }^{1}$ Universidade Federal Rural do Rio de Janeiro, Seropédica, Brasil

${ }^{2}$ Universidade Federal Fluminense, Volta Redonda, Brasil

\section{Article Info}

Palavras-chave:

Estratégia

Logística de Distribuição

Custo de Transporte

Submitted 25 Jun 2015;

received in revised form 5 Jul 2015;

accepted 12 Jul 2015

Licensed under

Creative Commons

CC-BY 3.0 BR.

\section{Resumo}

Distribuição Logística é uma importante vantagem competitiva quando a empresa atende a demanda de um cliente com menor custo. 0 presente estudo consiste em analisar o problema de alocação de clientes devido à inserção de novas unidades de fertilizantes nitrogenados no desenho da rede logística, minimizando o custo de transporte e, portanto reduzindo o custo do processo de distribuição do produto uréia. Através da aplicação do Problema Clássico de Transporte, foi realizada a revisão da rede logística realocando os clientes considerados estratégicos pela empresa XPTO à nova configuração da rede logística utilizando como critério o custo de transporte otimizado. Verificou-se que a realocação da demanda dos clientes estratégicos em função da aquisição da Unidade de Fertilizantes Nitrogenados no estado do Paraná proporcionou a mais significativa redução nos custos de transporte para a distribuição da uréia dentre os quatro cenários propostos. Portanto o trabalho corrobora com a teoria sobre a necessidade de revisar a rede logística quando novas instalações são inseridas na mesma.

+ Corresponding author. Rua Araguaia, 394. Jacarepaguá. Rio de Janeiro - RJ.

E-mail address: alessandraandradepereira@yahoo.com.br.

\section{Introdução}

Diante da busca por desempenho superior em um ambiente de concorrência, o alinhamento entre os níveis estratégicos, operacionais e funcionais de uma empresa tem importância na construção de vantagens competitivas sustentáveis e de longo prazo (RESENDE; MENDONÇA, 2007).

0 desdobramento da estratégia de negócios de uma empresa para os outros níveis deve estar alinhado para que não existam práticas desconectadas entre estes. Pois as decisões tomadas nos níveis operacionais e táticos devem suportar a estratégia competitiva da empresa.

Uma das principais decisões de uma empresa no nível estratégico é o Planejamento de Redes Logísticas que determina o número de instalações, tamanho e localização de fábricas e depósitos. 0 desdobramento desta decisão para o nível tático reflete na decisão de definir a alocação dos clientes às instalações (KOUVELIS, 1996).

Portanto quando uma nova instalação é introduzida na rede logística, a qual possui um lugar fixo, é necessário revisar a designação dos clientes às instalações de modo a minimizar o custo do transporte (JAYARAMAN; PIRKUL, 2001, JAYARAMAN; ROSS, 2003, MELO; NICKEL; SALDANHA-DA-GAMA, 2009, SYARIF ET AL., 2002).

Esse é o ponto de partida para o presente trabalho, um estudo de caso, cujo objetivo é analisar a realocação dos clientes às instalações fabris com a finalidade de minimizar os custos totais de transporte a partir das implantações das novas Unidades de Fertilizantes Nitrogenados (UFNs).

Através da aplicação do Problema Clássico de Transporte, pretendeu-se responder a seguinte questão: Quais clientes considerados estratégicos pela empresa deverão ser servidos por quais instalações para minimizar o custo de transporte?

Nesse sentido, o estudo procurou contribuir com maiores informações sobre a utilização do Problema Clássico de Transporte no processo de tomada de decisão, auxiliando o processo gerencial da empresa ao realocar os clientes à nova configuração da rede logística.

Para se atingir o proposto acima foi realizada a revisão bibliográfica sobre os temas Alinhamento Estratégico, Logística de Distribuição e o Papel do Transporte, além Localização de Instalações/Alocação de Demanda. 0 trabalho utilizou-se de um estudo exploratório do setor de fertilizantes brasileiro a partir de um estudo de caso apoiado por modelagem e simulações de cenários, com a proposta de realocação dos clientes nas novas UFNs. 


\section{Fundamentação teórica}

O alinhamento estratégico procura ajustar a organização com o seu ambiente e as decisões tomadas dentro da dimensão de cada sub-estratégia devem ser coerentes entre si e convergirem com a estratégia global de negócios (BORELLA; PADULA, 2010).

Para Mintzberg, Ahlstrand, e Lampel (2010), a lógica do alinhamento estratégico é não separar o pensamento da ação, a alta administração com a gerência intermediária, as pessoas que elaboram os planos e aqueles que os colocam em prática.

Para dar suporte à estratégia competitiva da empresa às estratégias de operações, que no caso de empresas industriais são considerados o fornecimento, a fabricação e a distribuição, deverão ir ao encontro das estratégias de negócios (WHEELWRIGHT, 1984).

A fim de se tornarem mais competitivas e eficientes, empresas tomam decisões no nível estratégico, como determinação do número, tamanho e localização das fábricas e depósitos, realocação de plantas e centros de distribuição, inclusão de novas instalações na rede logística, influenciando a Logística de Distribuição e acarretando mudanças em suas atividades.

A estratégia da Logística de Distribuição para Pagh e Cooper (1998) se destina a determinar a forma como a empresa irá atender o mercado e os clientes com os seus produtos e serviços. Portanto a logística de distribuição deve estar alinhada à estratégia de negócio da empresa e o transporte tem um papel importante neste processo. Pois para Ballou (2006) uma das atividades-chave que compõem a Logística de Distribuição é o Transporte. 0 transporte é uma das mais importantes áreas em matéria de planejamento de rede logística, em face do impacto que as decisões tomadas nela acabam tendo sobre a lucratividade, representando dentro dos custos logísticos, cerca de 60\%, tornando o produto menos competitivo no mercado.

Gurgel (2000) considera o Transporte como um elo essencial entre a expedição da empresa e o cliente, movimentando o produto entre a unidade produtiva e o cliente,

Para Chopra e Meindl (2010) o transporte exerce um papel crucial na logística de distribuição uma vez que os produtos raramente são fabricados e consumidos no mesmo local.

De acordo com Ballou (2006), o transporte pode ser utilizado como fator-chave se a estratégia de negócios da empresa for atender clientes que demandam um nível muito alto de responsividade. Por outro lado, se o preço for o principal critério de decisão de compra, o transporte pode ser utilizado para baixar o custo do produto.

Clientes distribuídos espacialmente, distâncias, tempo, custos entre clientes e instalações, quais as instalações devem ser abertas, quais clientes devem ser atendidos a partir de qual instalação de modo a minimizar os custos totais são questões envolvendo problemas de localização de instalações (do inglês, Facility Location) (MELO ET AL.; 2009, JAYARAMAN; ROSS, 2003)

Porém é necessário ter em mente os fatores estratégicos da empresa, pois estes exercem impactos nas decisões de projeto de rede dentro da cadeia de suprimento. Fatores tecnológicos, macroeconômicos, competitivos, tempo de resposta ao cliente e presença local, além dos custos de logística e instalações também são levados em consideração no momento de projetar a rede de instalações (CHOPRA; MEINDL, 2010).

Num segundo momento tem-se a otimização de rede de instalações através da alocação da demanda de mercados diferentes às instalações fabris com o objetivo de minimizar o custo total de instalação, estoque e transporte.

\section{Evolução do setor de fertilizantes no Brasil}

Durante todo o processo de expansão da agropecuária brasileira, o consumo de fertilizantes foi sustentado pelo aumento da importação. A dependência destes insumos agrícolas importados faz com que o Brasil se torne vulnerável às oscilações de mercado de fertilizantes, uma vez que a produção nacional das matérias-primas básicas dos fertilizantes (nitrogênio, fósforo e potássio) não acompanha o ritmo de crescimento da demanda (GONÇALVES; FERREIRA; SOUZA, 2008).

Dados da Associação Nacional para Difusão de Adubos - ANDA, em 2012, apontam que em torno de 67 por cento dos fertilizantes consumidos no Brasil foram importados (ANDA 2013).

Porém, recentemente o Ministério da Agricultura Pecuária e Abastecimento - MAPA propôs diminuir a dependência externa de matérias-primas pela agricultura brasileira, com maior produção interna, até o final de 2019.

Com relação aos fertilizantes nitrogenados, até 2017, apenas a empresa XPTO deverá investir em projetos de transformação do gás natural em amônia e uréia, com a implantação das chamadas UFNs (ANDA, 2013).

\section{Apresentação do estudo de caso}

A empresa XPTO é a maior produtora de fertilizantes nitrogenados do Brasil. Além de possuir duas fábricas no nordeste do país: a UFN-SE, com capacidade de produção de 657 mil toneladas/ano de uréia e 456 mil toneladas/ano de amônia e a UFN-BA, com 474 mil toneladas/ano de uréia e 474 mil toneladas/ano de amônia, a empresa reforçou a área de fertilizantes com a recente aquisição da UFN-PR no sul do país, com capacidade produtiva de 700 mil toneladas de uréia em Julho/2014.

Embora a estrutura e a operação da empresa estejam em andamento, pode se utilizar a técnica de alocação de demanda às instalações fabris para rever a sua logística de distribuição e alinhar a sua estrutura pré-existente às novas realidades do negócio.

Além disso, atualmente a empresa está investindo em novas unidades a fim de acompanhar o crescimento do mercado.

No centro-oeste, em Três Lagoas (MS) está sendo construída uma planta que entrará em operação em setembro de 2015 com capacidade para produzir 1,223 milhão de toneladas/ano de uréia e 70 mil toneladas/ano de amônia. No nordeste, município de Laranjeiras (SE), está sendo construída uma planta de sulfato de amônio com capacidade para produzir 303 mil toneladas/ano, que começará a produzir em 2016. Duas outras unidades no sudeste do Brasil, uma em Uberaba (MG) que produzirá 519 mil t/ano de amônia e outra em Linhares (ES), com produção de uréia (755 mil t/ano) e amônia (456 mil t/ano) têm previsão de início das operações em Setembro de 2016 e Junho de 2017 respectivamente.

0 estudo foi delimitado para o fertilizante nitrogenado Uréia, pois este produto representa $70 \%$ do faturamento da área de fertilizantes da empresa XPTO. De acordo com a ANDA (2013), a Uréia tem seu consumo maior do que os outros 
fertilizantes nitrogenados, principalmente pela maior percentagem do nutriente Nitrogênio e pelo menor custo de frete em relação à quantidade de Nitrogênio transportada.

Devido aos novos projetos das UFNs é necessária uma reavaliação da logística de distribuição do fertilizante nitrogenado uréia para os quatro (4) clientes considerados estratégicos pela empresa XPTO, responsáveis por demandar 55,8\% da uréia produzida. Uma vez que, até Julho de 2014 os clientes estratégicos eram designados às instalações localizadas no nordeste do país, estados da Bahia e Sergipe.

O cliente A possui 21 destinos, com demanda média anual de 13.901,21 toneladas de uréia/destino. 0 cliente B possui 13 destinos, com demanda média anual de 9.364,55 toneladas de uréia/destino. 0 cliente $\mathrm{C}$ possui 05 destinos, com demanda média anual de 9.192,55 toneladas de uréia/destino. E o cliente D possui 05 destinos, com demanda média anual de 7.204,97 toneladas de uréia/destino.

\section{Método de pesquisa}

O método adotado é classificado como uma pesquisa científica de natureza aplicada, seguindo abordagem quantitativa, com objetivos exploratórios. Utiliza-se do estudo de caso para contextualizar a aplicação do problema e simulação como procedimento técnico. (BERTRAND; FRANSO0, 2002).

O Problema Clássico de Transporte é o mais representativo dos Problemas de Programação Linear, de grande aplicação prática e tem como objetivo determinar as quantidades de produtos a serem transportadas a partir de um conjunto de fornecedores para um conjunto de consumidores, de forma que o custo total de transporte seja minimizado. 0 problema considera dois elos da cadeia de suprimentos e não incluem instalações intermediárias, como centros de distribuição (BELFIORE; FÁVERO, 2012).

Função Objetivo:

$\min z=\sum_{i=1}^{m} \sum_{j=1}^{n} c_{i j} x_{i j}(1)$

Sujeito a:

$$
\begin{array}{ll}
\sum_{j=1}^{n} x_{i j} \leq C f_{i}, & i=1,2, \ldots, m \\
\sum_{i=1}^{m} x_{i j} \geq d_{j}, & j=1,2, \ldots, n \\
x_{i j} \geq 0, & i=1,2, \ldots, m, \quad j=1,2, \ldots, n
\end{array}
$$

Em que:

Cij = custo unitário de transporte do fornecedor i para o consumidor $\mathrm{j}$

$\mathrm{Xij}=$ quantidades transportadas do fornecedor i para o consumidor $\mathrm{j}$

$\mathrm{Cfi}=$ capacidade de abastecimento do fornecedor $\mathrm{i}(\mathrm{i}=1, \ldots, \mathrm{m})$

$\mathrm{dj}=$ demanda do destino $\mathrm{j}(\mathrm{j}=1, \ldots, \mathrm{n})$

A equação 1 representa a função objetivo do modelo que visa minimizar o custo de transporte em função das distâncias entre os pontos; instalações e clientes. Com relação às restrições vale ressaltar que a Equação 2 é a restrição de limite de capacidade de fornecimento e a Equação 3 representa a restrição de cobertura de demanda, na qual todos os clientes deverão ter o total de suas demandas atendidas. A Equação 4 é a restrição de não-negatividade.

Através do Anuário 2012 do Sistema de Informações de Fretes (SINFRECA), principal referência do mercado de fretes agrícolas no país, foi obtido o valor médio de 0,1091R \$/t.km para adubos e fertilizantes no ano de 2012. Esse valor foi multiplicado pela distância entre os pontos de origens das fábricas e destinos dos clientes estratégicos, obtidos através do Google Maps que é considerado uma ferramenta de Sistemas de Informação Geográfica, resultando no custo de transporte. Levantadas as variáveis acima, o Problema Clássico de Transporte foi resolvido por meio de a ferramenta Solver da planilha eletrônica MS Excel®.

\section{Simulação}

Definem-se os cenários considerando as mudanças na rede logística devido às inserções de novas instalações na área de fertilizantes da empresa XPTO. É importante destacar que foi considerada a demanda fictícia Dummy em todos os cenários para balancear o problema Clássico de Transporte, isto é, igualar demanda e oferta.

Para o cenário 1 as quantidades ofertadas pelas UFN-SE e UFN-BA, 580 tons e 375 tons respectivamente, foram distribuídas entre os quatro clientes (A, B, C e D) e a variável Dummy, totalizando 33 destinos, visando minimizar o custo de transporte através da redução das distâncias entre os pontos de instalações e clientes.

A restrição de limite de capacidade de fornecimento foi respeitada uma vez que o volume demandado pelos 4 clientes não ultrapassou a capacidade de fornecimento, incluindo a variável Dummy como cliente/destino para igualar oferta e demanda. E a restrição de cobertura de demanda também foi respeitada, pois todos os clientes tiveram o total de suas demandas atendidas.

À medida que novas fábricas são inseridas na rede logística devido ao plano estratégico da empresa, os 33 destinos são realocados considerando a rede logística anterior. Portanto no Cenário 2, a quantidade ofertada de uréia passa a ser de 1,375 tons devido à inserção de 420 tons ofertada pela UFN-PR. Neste cenário aumenta a quantidade de toneladas destinada 
à variável Dummy e realoca a demanda dos 33 destinos considerando o menor custo de transporte. 0 mesmo procedimento é realizado para os cenários 3 e 4 com as inserções das UFNs III e IV com o aumento de 927 tons e 572 tons respectivamente.

\section{Análise dos resultados}

A função objetivo de todos os cenários foi a de minimizar o custo de transporte. Os resultados numéricos dos cenários gerados pelo Solver podem ser obtidos na tabela 1 .

A solução Solver apresentada para o cenário 1 menciona o custo de transporte otimizado no valor de R \$ 92.798.672,78 para distribuir a uréia a partir das fábricas localizadas no nordeste do país (nos estados da BA e SE) aos 4 clientes estratégicos. 0 fornecimento para os 32 pontos de demanda, considerando a aquisição da planta no sul do país, reduziu o custo de transporte aos clientes estratégicos em torno de $53,7 \%$ de acordo com o cenário 2 . No cenário 3 , a construção de uma fábrica na região centro-oeste do país reduziu o custo de transporte em $27,7 \%$ comparado ao cenário 2 . A redução do custo de transporte para os clientes estratégicos no cenário 4 não foi significativa comparada aos cenários anteriores.

Table 1 - Soluções geradas para os cenários simulados

\begin{tabular}{lllll}
\hline Cenários & $\begin{array}{l}\text { Características } \\
\text { Fábricas Existentes }\end{array}$ & Novas Fábricas & Total de Fábricas & Custo de Transporte (R\$) \\
\hline 1 & 2 & - & 2 & $92.798 .672,78$ \\
2 & 2 & 1 & 3 & $42.937 .264,30$ \\
3 & 3 & 1 & 4 & $31.041 .808,71$ \\
4 & 4 & 1 & 5 & $27.418 .076,59$ \\
\hline
\end{tabular}

Pela análise da tabela 3, pode-se perceber que os valores de custo de transporte aos clientes estratégicos diminuem à medida que se aumenta o número de fábricas, uma vez que assim os clientes ficam mais próximos, reduzindo o custo de transporte.

Verificou-se que a realocação da demanda dos clientes estratégicos em função da aquisição da UFN-PR proporcionou a mais significativa redução nos custos de transporte para a distribuição da uréia dentre os quatro cenários propostos.

A otimização do custo de transporte na logística de distribuição da uréia para os clientes estratégicos pode ser considerada um exemplo de alinhamento estratégico para a área de fertilizantes da XPTO podendo gerar melhoras nos processos de entrega e maximização da margem do produto.

\section{Conclusão}

A partir da aplicação prática do Problema Clássico de Transporte no estudo de caso foi possível analisar as inserções de novas UFNs na rede logística da empresa XPTO e o impacto na realocação da demanda dos clientes estratégicos, considerando o critério custo de transporte.

Deve ser levado em consideração que a abordagem do trabalho se restringiu somente ao custo de transporte, não sendo considerados outros custos neste trabalho. Porém para o fertilizante o custo de transporte é muito significativo. Por se tratar de uma commodity a uréia não apresenta diferenciação e possui baixo valor agregado, fator que eleva a participação do custo de transporte na formação do seu preço, soma-se a isso a localização da demanda distante dos pontos de oferta, o volume transportado e a falta de isonomia tributária entre o produto nacional e o importado faz com que o custo de transporte se torne uma variável importante.

Portanto, o alcance dos objetivos se deu a partir dos resultados gerados pela aplicação do Problema Clássico de Transporte em simulações de cenários. Constatou-se que, à medida que se insere uma nova UFN na rede logística da XPTO originando a necessidade de revisão na mesma, a demanda de uréia pelos clientes estratégicos é realocada diminuindo os custos de transporte. A aquisição da UFN-PR providenciou a maior redução no custo de transporte para os clientes estratégicos.

Por fim, dada a natureza do estudo de caso, é possível considerar que a realocação da demanda em um Planejamento de Redes Logísticas é um importante instrumento capaz de contribuir com a otimização na distribuição do produto, reduzindo o custo de transporte.

Com relação às limitações da pesquisa, a consideração das previsões das capacidades de oferta para as unidades de fertilizantes nitrogenados que estão em fase de construção/projeto pode ser uma limitação, visto que as mesmas poderão não estar de acordo com a realidade destas quando em operação.

Com relação à demanda, embora os 4 clientes sejam responsáveis por demandar cerca de $60 \%$ da uréia produzida pela XPTO, a não consideração dos demais clientes caracteriza uma limitação deste estudo.

Outras limitações apontadas são: a não consideração dos custos totais, a consideração exclusiva do transporte rodoviário em detrimento de outros modos de transporte, o estabelecimento de um valor médio do frete levando a omissão das variações nos custos de transportes tais como condições de vias, pedageamento e frete de retorno, por exemplo, bem como a não consideração de aspectos tributários, pois estes geram impactos na rede logística.

Com base nas limitações acima citadas, emergem como aspectos a serem trabalhados em estudos futuros: a correta informação sobre a capacidade de oferta das unidades de fertilizantes nitrogenados que estão em fase de construção/projeto; a consideração de todo o portfólio de clientes; a consideração de diferentes modos de transporte como alternativa para a distribuição da uréia, como por exemplo, hidroviário, a consideração de outras variáveis relevantes para o cálculo dos custos efetivos de transportes de modo mais amplo, como combustível, mão-de-obra, depreciação, manutenção, 
seguro do veículo, entre outras e a consideração da estrutura do ICMS, que está associado ao planejamento de redes logísticas.

\section{References}

ASSOCIAÇ̃̃ NACIONAL PARA DIFUSÃO DE ADUBOS - ANDA. (2013). Setor de Fertilizantes - Anuário Estatístico 2012.

BALLOU, R. H. (2006). Gerenciamento da Cadeia de Suprimentos / Logística Empresarial. Porto Alegre: Bookman.

BELFIORE, P., \& FÁVEROL. P. (2012). Pesquisa Operacional Para cursos de Administração, Contabilidade e Economia. Rio de Janeiro: Elsevier.

BERTRAND, J. W. M., \&FRANSOO, J. C. (2002).Modelling and simulation: operations management research methodologies using quantitative modeling. International Journal of Operations \& Production Management, volume ( 22), n. 2, p.241-264.

BORELLA, M. R. de C.\& PADULA, A. D. (2010). Alignment Between The Supply, Manufacturing And Distribution Strategies And The Business Strategy. Journal of Operations and Supply Chain Management, volume (3), n. 2, p. $44-60$.

CHOPRA, S.; MEINDL, P. (2010). Gerenciamento da Cadeia de Suprimentos - Estratégia, Planejamento e Operação. São Paulo: Pearson.

GONÇALVES, J. S., FERREIRA, C. R. R. P. T.\& SOUZA, S. A. M. (2008). Produção nacional de fertilizantes, processo de desconcentração regional e maior dependência externa. Informações Econômicas, volume.38, Agosto.

GURGEL, F. A.(2000).Logística Empresarial. São Paulo: Atlas.

JAYARAMAN, V.\&PIRKUL, H. (2001).Planning and coordination of production and distribution facilities for multiple commodities. European Journal of Operational Research. volume.133, n. (2), p.394-408.

JAYARAMAN, V.; ROSS, A. (2003). A simulated annealing methodology to distribution network design and management. European Journal of Operational Research, Vol.144, n.3. p. 629-645.

KOUVELIS, P.; CHAMBERS, C. \&WANG, H. (2006). Supply Chain Management Research and Production and Operations Management: review, trends and opportunities. Production and Operations Management, volume. (15), n. 3, p.449- 469.

MELO, M. T.; NICKEL, S. \& SALDANHA-DA-GAMA, F. (2009). Facility location and supply chain management - A review.European Journal of Operational Research, volume (196), n.2, p. 401-412.

MINTZBERG, H.; AHLSTRAND, B. \& LAMPEL, J. (2010).SAFARI DE ESTRATÉGIA. UM ROTEIRO PELA SELVA DO PLANEJAMENTO ESTRATÉGICO. Porto Alegre Bookman.

PAGH, J. D.; COOPER, M. C. (1998). Supply Chain Postponement and Speculation Strategies: How to Choose the Right Strategy. Journalof Business Logistics, volume(19), n. 2, p. $13-33$.

RESENDE, P. T. V.\& MENDONÇA, G. D. Práticas de Gestão de Estoques, Armazenagem e Transporte nos Canais de Distribuição Brasileiros. XXXI Encontro da ANPAD. Rio de Janeiro, Brasil.

SYARIF, A.; YUN, Y. \& GEN, M. (2002). Study on multi-stage logistic chain network: a spanning tree-based genetic algorithm approach. Computers\& Industrial Engineering, volume (43), n.1-2, p. 299-314.

SISTEMA DE INFORMAC̄ÕES DE FRETES - SIFRECA. Anuário 2012.

WHEELWRIGHT, S. C. Manufacturing Strategy: defining the missing link. Strategic Management Journal, vol. 5, n.1, p.77 - 91.1984.

\section{Abstract}

Distribution Logistics is an important competitive advantage when the company serves customers demands with lower cost. The present study aims to analyze the problem of customers' allocation due the insertion of new nitrogen fertilizers units in the logistic networking desing, minimizing transportation costs and hence, in the product's distribution process of urea. by applying the Classic Transport Problem was realized the review of logistics network relocating customers considered strategic by the company XPTO to the new configuration of the logistics network using as criteria the costoptimized transport. It was found that the relocation of demand from strategic customers due to the acquisition of Nitrogenous Fertilizer Unit in Parana state provided the most significant reduction in transportation costs for the distribution of urea among the four proposed scenarios. So the work confirms the theory that is necessary to review the logistics network when new facilities are inserted in it.

Key words: strategy, distribution logistics, transportation costs. 